\title{
Dynamic model of a railway wheelset for corrugation problem analysis
}

\author{
Paloma Vila ${ }^{1}$, Andrés Rovira ${ }^{1}$, Juan Fayos ${ }^{2}$ and Luis Baeza ${ }^{1 *}$ \\ ${ }^{1}$ Dpto. de Ingeniería Mecánica, Universidad Politécnica de Valencia, Spain \\ 2Instituto de Biomecánica de Valencia, Universidad Politécnica de Valencia, Spain \\ *email: Ibaeza@mcm.upv.es
}

\begin{abstract}
In the present work a model of flexible shafts is adapted for modelling the railway wheelset. The dynamic properties of the solid are obtained from a solid finite element model of a real wheelset. The model adopts an Eulerian coordinate set for numerical efficiency. Results of the crossedreceptance and mobility functions are presented, where the excitation is the vertical wheel-rail contact force and the displacement (or velocity) is measured in the wheel-rail contact plane. These results let us know that the gyroscopic effect may contribute to define the wavelength-fixing mechanism in some corrugation problems, even if the train speed is low.
\end{abstract}

\section{Introduction}

The conventional modelling of the railway vehicle, in which it is considered as a system of rigid bodies connected by viscoelastic elements, yields accurate enough results in the low-frequency range (up to $20 \mathrm{~Hz}$, approximately). However, when it comes to analysing the dynamic interaction of the vehicle and the track in the mid- and highfrequency ranges (when studying problems such as corrugation) the use of these simplified wheelset models may lead to inaccurate results. This is due to the fact that in these frequency ranges the structural dynamics of the wheelset cannot be neglected. Accordingly, in these cases a more realistic wheelset model able to reproduce the elastic behaviour of the rotating wheelset should be adopted.

There exist a few research works in which the flexibility of the wheelset and the effects associated with rotation are considered. One of the pioneering works can be found in Ref. [1], in which the contact force is considered as a moving load on the perimeter of the wheel. In that work, only one wheel is modelled as a disc, and the results show that each peak at the FRF function is decoupled into two resonances. However, the inertial effects due to rotation are neglected. The role of the inertial effects due to rotation in beams is discussed in [2], where it is found that these effects are more significant if the slenderness of the beam is low, and that is the case of the railway wheelset.

A model that considers the gyroscopic dynamics is due to Szolc [3-5], who considers a non-rotating beam (wheelset axle) linked through springs to rotating rigid bodies that represent the ring-wheels and brake discs. It is stated that the modal properties of the nonrotating wheelset differs from those of the rotating one. A similar approach was done in [6], where the dynamics of wheelsets that mount elastic wheels is studied.

The modelling of the continuous system can be based on general procedures of flexible multibody systems such as the method presented in [7-8], which uses modal coordinates, or through the Finite Element (FE) Method. These methods are based on Lagrangian coordinates and their main difficulty is the interaction with non-rotating structures, such as the track and the axleboxes in the case of the wheelset. The position of the contact point is occupied by a different material point in each time instant and consequently, the computational cost increases.

A continuous system approach of the wheelset was carried out in [9-10]. The method adopts a FE model and considers the inertial forces due to rotation. In order to solve the problems related to the interaction with the track, the contact forces were supposed to be applied in the centre axle of the wheelset.

An alternative to the previous methods is the use of Eulerian coordinates. The first works that show results from this methodology correspond to the Refs. [11-14]. Nevertheless, they did not publish their method until 2007 in Ref. [15].

Simultaneously, a method that employs Eulerian coordinates was published in [16]. The techniques are, however, very different:

This paper was presented at ISMA 2008, and is reproduced here with the kind permission of the conference chairman, Professor Sas, and the authors. 
the former can be applied to solids that have angular periodicity; the latter exploits the modal properties of solids of revolution.

The present work studies the potential of using a flexible wheelset model which implements the inertial effects due to rotation for analysing the rail corrugation problem. Section 3 shows results that establish the influence of the rotation on the contact point displacements when a harmonic vertical force is applied in the contact points of the wheels. The wheelset model employed in the calculations is based on the method presented in Ref. [16]. The technique is summarised in Subsection 2.2 of the present article. The background of the method is the formulation developed by Shabana and presented in [7]. In order to make clear the method based on Eulerian coordinates, the needed formulas from [7] are shown in Subsection 2.2.

\section{Equations of motion development 2.1 General method for flexible bodies based on Lagrangian coordinates}

Let us consider a flexible wheelset rotating about its main axis at constant angular velocity, $\Omega$. Two reference systems are adopted, one fixed (non-rotating) and the other rotating with the undeformed wheelset. Both coincide at the initial time. Any particle vector position $\overrightarrow{\mathbf{r}}$ can be written through the vector position of the particle in the undeformed body $\overrightarrow{\mathrm{u}}$, and the particle displacement due to deformation $\overrightarrow{\mathbf{u}}_{f}$ as follows (see Figure 1)

$$
\overrightarrow{\mathbf{r}}=\overrightarrow{\mathbf{u}}+\overrightarrow{\mathbf{u}}_{f}
$$

The matrix expressions of the components of the previous equation have different values depending on whether they are expressed in the rotating $\left(\mathbf{r}^{\prime}, \mathbf{u}^{\prime}\right.$ and $\left.\mathbf{u}_{f}^{\prime}\right)$ or the non-rotating frame $\left(\mathbf{r}, \mathbf{u}\right.$ and $\left.\mathbf{u}_{f}\right)$. The transformation matrix lets us obtain the relation between these expressions as follows

$$
\mathbf{r}\left(\mathbf{u}^{\prime}, t\right)=\mathbf{A} \mathbf{r}^{\prime}=\mathbf{A}(t)\left(\mathbf{u}^{\prime}+\mathbf{u}_{f}^{\prime}\left(\mathbf{u}^{\prime}, t\right)\right) .
$$

If small deformations of the solid are considered, the deformed shape of the solid can be obtained as a combination of the nonrotating mode shapes. If the modal approach is adopted, Eq. (2) can be written as follows

$$
\mathbf{r}\left(\mathbf{u}^{\prime}, t\right)=\mathbf{A} \mathbf{r}^{\prime}=\mathbf{A}(t)\left(\mathbf{u}^{\prime}+\boldsymbol{\Phi}\left(\mathbf{u}^{\prime}\right) \mathbf{p}(t)\right),
$$

where $\boldsymbol{\Phi}\left(\mathbf{u}^{\prime}\right)$ is the mass-normalised matrix of the mode shapes of the non-rotating wheelset, and $\mathbf{p}(t)$ contains the respective modal coordinates. This kinematic approach permits

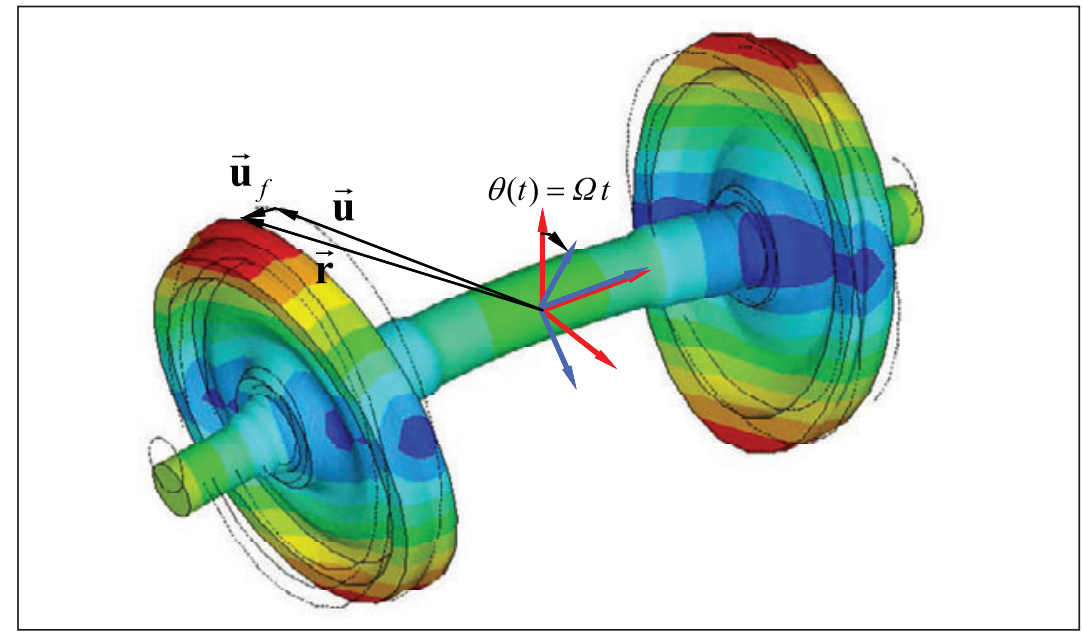

Figure 1. Kinematic relationships.

to obtain the following equation of motion

$$
\ddot{\mathbf{p}}+2 \Omega \tilde{\mathbf{J}} \dot{\mathbf{p}}+\left(\widetilde{\mathbf{K}}-\Omega^{2} \widetilde{\mathbf{E}}\right) \mathbf{p}=\mathbf{Q}_{\mathbf{p}}+\Omega^{2} \tilde{\mathbf{L}}
$$

where $\tilde{\mathbf{K}}$ is a diagonal matrix that contains the square of the natural frequencies of the nonrotating wheelset, and the rest of components of Eq. (4) are

$$
\begin{gathered}
\widetilde{\mathbf{J}}=\int_{\text {Volume }} \rho \boldsymbol{\Phi}^{\mathrm{T}} \mathbf{J} \boldsymbol{\Phi} \mathrm{d} v, \quad \mathbf{J}=\frac{1}{\Omega} \mathbf{A}^{\mathrm{T}} \dot{\mathbf{A}}, \\
\widetilde{\mathbf{E}}=\int_{\text {Volume }} \rho \boldsymbol{\Phi}^{\mathrm{T}} \mathbf{E} \boldsymbol{\Phi} \mathrm{d} v, \quad \mathbf{E}=\frac{-1}{\Omega^{2}} \mathbf{A}^{\mathrm{T}} \ddot{\mathbf{A}},
\end{gathered}
$$

$$
\widetilde{\mathbf{L}}=\int_{\text {Volume }} \rho \boldsymbol{\Phi}^{\mathrm{T}} \mathbf{E} \mathbf{u}^{\prime} \mathrm{d} v
$$

It must be highlighted that the matrices $\tilde{\mathrm{J}}, \widetilde{\mathrm{E}}$ and $\tilde{\mathbf{L}}$ are constant in spite of the expressions of matrices $\mathrm{J}$ and $\mathrm{E}$. Taking into account that the solid motion is a rotation around a constant axis, then the matrices $\mathrm{J}$ and $\mathrm{E}$ are constant and it is satisfied that

$\mathbf{J}=\frac{1}{\Omega} \mathbf{A}^{\mathrm{T}} \dot{\mathbf{A}}=\frac{1}{\Omega} \dot{\mathbf{A}} \mathbf{A}^{\mathrm{T}} \quad$ and $\quad \mathbf{E}=\frac{-1}{\Omega^{2}} \mathbf{A}^{\mathrm{T}} \ddot{\mathbf{A}}=\frac{-1}{\Omega^{2}} \ddot{\mathbf{A}} \mathbf{A}^{\mathrm{T}}$.

The external forces $\mathbf{f}$ are assumed to be written as a product of the spatial and time dependent functions, that is $\mathbf{f}\left(\mathbf{u}^{\prime}, t\right)=\mathbf{f}_{u}\left(\mathbf{u}^{\prime}\right) \mathbf{f}_{t}(t)$. The generalised force vector $Q_{p}$ has the following form

$$
\begin{aligned}
& \mathbf{Q}_{\mathbf{p}}=\int_{\text {Volume }} \frac{\partial \mathbf{r}^{\mathrm{T}}\left(\mathbf{u}^{\prime}, t\right)}{\partial \mathbf{p}} \mathbf{f}\left(\mathbf{u}^{\prime}, t\right) \mathrm{d} v=\int_{\text {Volume }} \boldsymbol{\Phi}^{\mathrm{T}}\left(\mathbf{u}^{\prime}\right) \mathbf{A}(t)^{\mathrm{T}} \mathbf{f}\left(\mathbf{u}^{\prime}, t\right) \mathrm{d} v \\
& =\left[\int_{\text {Volume }} \boldsymbol{\Phi}^{\mathrm{T}}\left(\mathbf{u}^{\prime}\right) \mathbf{A}(t)^{\mathrm{T}} \mathbf{f}_{u}\left(\mathbf{u}^{\prime}\right) \mathrm{d} v\right] \mathbf{f}_{t}(t) .
\end{aligned}
$$


The formulation of the generalised force vector involves the following difficulties inherent to the method:

- The expression into square brackets is not constant and must be computed for each time instant of the ODEs integration process, thus increasing the simulation time.

Taking into consideration that the mode shapes are obtained numerically, a constant force in the contact point produces a variable generalised force because of the numerical errors in the computation of the mode shapes.

Accordingly, a numerical excitation is derived.

\subsection{Method based on Eulerian coordinates}

The general method presented in the previous Subsection involves numerical and computational problems if the rotating solid interacts with a non-rotating structure. In order to solve these problems, an Eulerian approach is proposed. In railway wheelset dynamics we centre our attention on spatial points (i.e. the wheel-rail contact point). However, a spatial point is not occupied by an only material point. The coordinate of a spatial point in the undeformed state $\mathbf{u}$ will be occupied by material points whose coordinates depend on time

$$
\mathbf{u}^{\prime}(t)=\mathbf{A}(t)^{\mathrm{T}} \mathbf{u} .
$$

From (3), the final position of the point that occupies the spatial position $\mathbf{u}$ is

$$
\mathbf{r}\left(\mathbf{A}(t)^{\mathrm{T}} \mathbf{u}, t\right)=\mathbf{u}+\mathbf{A}(t) \boldsymbol{\Phi}\left(\mathbf{A}(t)^{\mathrm{T}} \mathbf{u}\right) \mathbf{p}(t) .
$$

Finally, the Eulerian position vector is defined as follows

$$
\mathbf{s}(\mathbf{u}, t)=\mathbf{u}+\mathbf{A}(t) \boldsymbol{\Phi}\left(\mathbf{A}(t)^{\mathrm{T}} \mathbf{u}\right) \mathbf{p}(t) .
$$

Solids with revolution geometry (such as a wheelset) have two families of vibration modes: modes with multiplicity 1 and modes with multiplicity 2 . The formers observe that the deformed geometry of the mode have revolution geometry (see Figure 2). The latters are associated with a couple of identical deformed mode shapes in two planes (usually orthogonal planes) which contain the rotation axis (see Figure 3). It is assumed that any deformed geometry of the solid can be obtained as a modal combination of the nonrotating mode shapes in the non-rotating frame. It can be mathematically expressed as follows

$$
\mathbf{s}(\mathbf{u}, t)=\mathbf{u}+\boldsymbol{\Phi}(\mathbf{u}) \mathbf{q}(t),
$$

being $\mathbf{q}(t)$ a set of Eulerian modal coordinates.

The Eulerian to Lagrangian transformation can be defined as follows

$$
\mathbf{q}(t)=\mathbf{B}(t) \mathbf{p}(t) .
$$

Matrix B can be proved to be orthogonal and quasi-diagonal. A detailed analysis shows that rows and columns associated with modes with multiplicity 1 have null entries except the diagonal entry which is 1 . On the other hand, diagonal terms associated with modes with multiplicity 2 contain rotation matrices, i.e.:

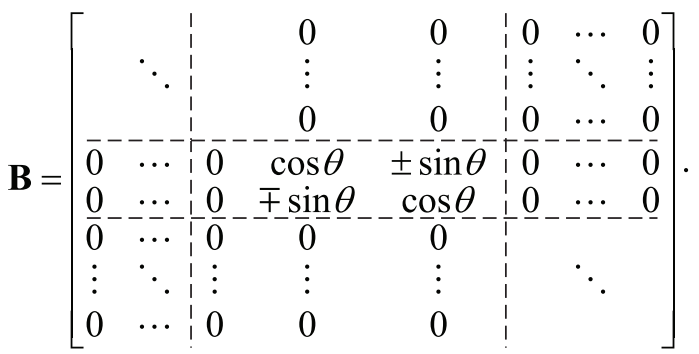

By comparing Eqs. (12) and (13), the next relationship is fulfilled

$$
\mathbf{A}(t) \boldsymbol{\Phi}\left(\mathbf{u}^{\prime}\right)=\boldsymbol{\Phi}\left(\mathbf{A}(t) \mathbf{u}^{\prime}\right) \mathbf{B}(t) .
$$

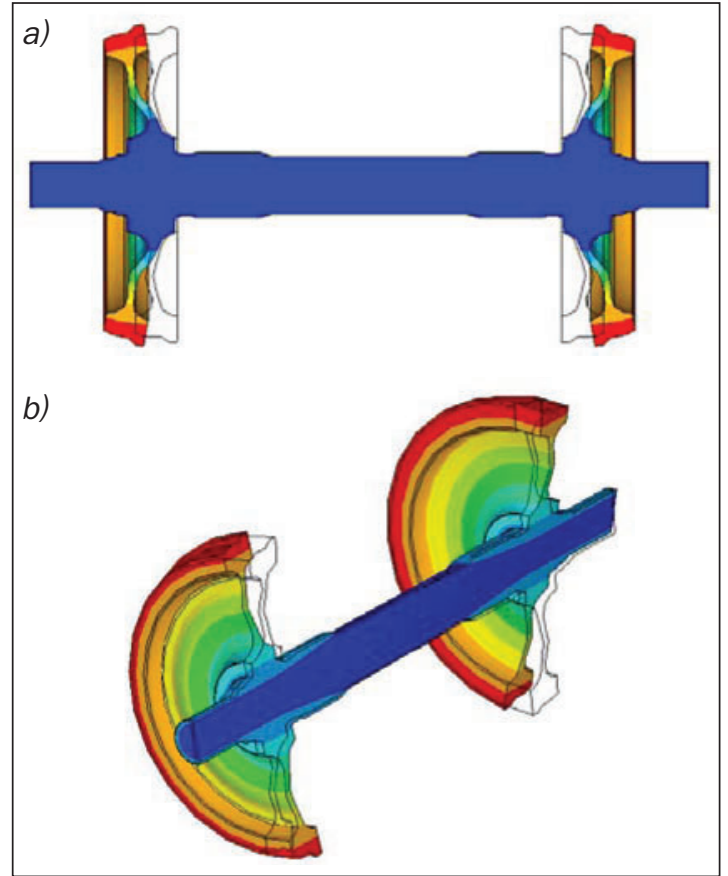

Figure 2. Examples of single multiplicity vibration modes of the non-rotating wheelset. a) Umbrella mode. b) First torsional mode. 


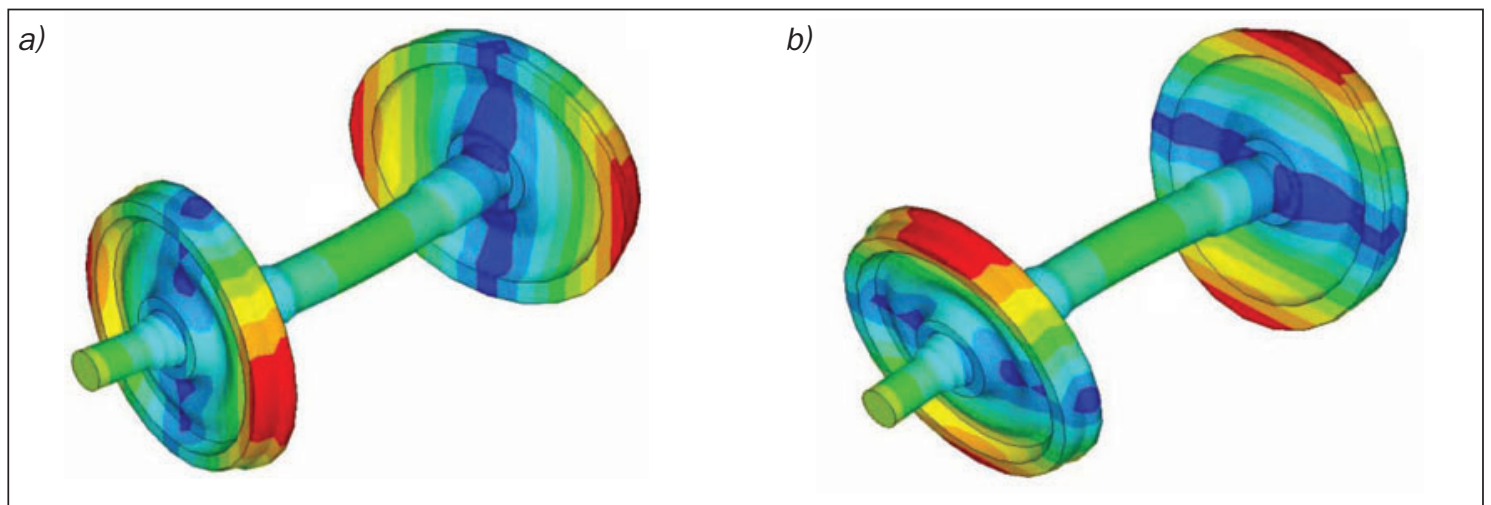

Figure 3. Examples of double multiplicity vibration modes of the non-rotating wheelset. a) First bending mode, horizontal plane. b) First bending mode, vertical plane.

If Eq. (16) is pre-multiplied by $\mathbf{A}(t)^{\mathrm{T}}$ and post-multiplied by $\mathbf{B}(t)^{\mathrm{T}}$, the last expression is equivalent to

$$
\mathbf{A}(t)^{\mathrm{T}} \boldsymbol{\Phi}\left(\mathbf{A}(t) \mathbf{u}^{\prime}\right)=\boldsymbol{\Phi}\left(\mathbf{u}^{\prime}\right) \mathbf{B}(t)^{\mathrm{T}} .
$$

The original equation of motion (4) can be written through Eulerian modal coordinates, giving

$$
\begin{gathered}
\ddot{\mathbf{q}}+\left(2 \Omega \mathbf{B} \widetilde{\mathbf{J}} \mathbf{B}^{\mathrm{T}}+2 \mathbf{B} \dot{\mathbf{B}}^{\mathrm{T}}\right) \dot{\mathbf{q}}+ \\
+\left(\mathbf{B} \widetilde{\mathbf{K}} \mathbf{B}^{\mathrm{T}}-\Omega^{2} \mathbf{B} \widetilde{\mathbf{E}} \mathbf{B}^{\mathrm{T}}+2 \Omega \mathbf{B} \widetilde{\mathbf{J}} \dot{\mathbf{B}}^{\mathrm{T}}+\mathbf{B} \ddot{\mathbf{B}}^{\mathrm{T}}\right) \mathbf{q} \\
=\mathbf{B} \mathbf{Q}_{\mathbf{p}}+\Omega^{2} \mathbf{B} \widetilde{\mathbf{L}} .
\end{gathered}
$$

The transformation matrix $\mathbf{B}$ does not change the numerical value of $\widetilde{\mathbf{J}}$ as is proved in the following result. From Eq. (5)

$$
\begin{gathered}
\mathbf{B} \widetilde{\mathbf{J}} \mathbf{B}^{\mathrm{T}}=\mathbf{B} \int_{\text {Volume }} \rho \boldsymbol{\Phi}\left(\mathbf{u}^{\prime}\right)^{\mathrm{T}} \mathbf{J} \Phi\left(\mathbf{u}^{\prime}\right) \mathrm{d} v \mathbf{B}^{\mathrm{T}}= \\
=\frac{1}{\Omega} \int_{\text {Volume }} \rho \mathbf{B} \boldsymbol{\Phi}\left(\mathbf{u}^{\prime}\right)^{\mathrm{T}} \mathbf{A}^{\mathrm{T}} \dot{\mathbf{A}} \boldsymbol{\Phi}\left(\mathbf{u}^{\prime}\right) \mathbf{B}^{\mathrm{T}} \mathrm{d} v .
\end{gathered}
$$

By substituting (17) in (19), and taking into account (8)

$$
\begin{gathered}
\mathbf{B} \widetilde{\mathbf{J}} \mathbf{B}^{\mathrm{T}}=\frac{1}{\Omega} \int_{\text {Volume }} \rho \boldsymbol{\Phi}\left(\mathbf{A} \mathbf{u}^{\prime}\right)^{\mathrm{T}} \mathbf{A} \mathbf{A}^{\mathrm{T}} \dot{\mathbf{A}} \mathbf{A}^{\mathrm{T}} \boldsymbol{\Phi}\left(\mathbf{A} \mathbf{u}^{\prime}\right) \mathrm{d} v= \\
\frac{1}{\Omega} \int_{\text {Volume }} \rho \boldsymbol{\Phi}\left(\mathbf{A} \mathbf{u}^{\prime}\right)^{\mathrm{T}} \dot{\mathbf{A}} \mathbf{A}^{\mathrm{T}} \boldsymbol{\Phi}\left(\mathbf{A} \mathbf{u}^{\prime}\right) \mathrm{d} v= \\
\int_{\text {Volume }} \rho \boldsymbol{\Phi}\left(\mathbf{A} \mathbf{u}^{\prime}\right)^{\mathrm{T}} \mathbf{J} \boldsymbol{\Phi}\left(\mathbf{A} \mathbf{u}^{\prime}\right) \mathrm{d} v
\end{gathered}
$$

If the change of variable $\mathbf{u}=\mathbf{A} \mathbf{u}^{\prime}$ is carried out (the Jacobian $|\mathbf{A}|$ is 1), Eq. (20) becomes (note that the integration variable is a dummy variable)

$$
\mathbf{B} \widetilde{\mathbf{J}} \mathbf{B}^{\mathrm{T}}=\frac{1}{\Omega} \int_{\text {Volume }} \rho \boldsymbol{\Phi}(\mathbf{u})^{\mathrm{T}} \mathbf{J} \boldsymbol{\Phi}(\mathbf{u}) \mathrm{d} v=\widetilde{\mathbf{J}} .
$$

In the same way, the next equivalences can be proved

$$
\begin{gathered}
\mathbf{B} \widetilde{\mathbf{L}}=\widetilde{\mathbf{L}}, \quad \mathbf{B} \widetilde{\mathbf{K}} \mathbf{B}^{\mathrm{T}}=\widetilde{\mathbf{K}}, \quad \mathbf{B} \widetilde{\mathbf{E}} \mathbf{B}^{\mathrm{T}}=\widetilde{\mathbf{E}}, \\
\mathbf{B} \dot{\mathbf{B}}^{\mathrm{T}}=\Omega(\widetilde{\mathbf{J}}+\widetilde{\mathbf{G}}) \\
\mathbf{B} \ddot{\mathbf{B}}^{\mathrm{T}}=-\Omega^{2}(\widetilde{\mathbf{J}}+\widetilde{\mathbf{G}})\left(\widetilde{\mathbf{J}}^{\mathrm{T}}+\widetilde{\mathbf{G}}^{\mathrm{T}}\right),
\end{gathered}
$$

being $\widetilde{\mathrm{G}}$ the gyroscopic matrix defined as

$$
\widetilde{\mathbf{G}}=\int_{\text {Volume }} \rho\left(\sum_{i=1}^{3} \frac{\partial \boldsymbol{\Phi}\left(\mathbf{u}^{\prime}\right)^{\mathrm{T}}}{\partial u_{i}}\left(\mathbf{J} \mathbf{u}^{\prime}\right)_{i}\right) \boldsymbol{\Phi}\left(\mathbf{u}^{\prime}\right) d v .
$$

Thus, replacing from (22) to (25) in (18), the equation of motion in Eulerian coordinates results

$$
\ddot{\mathbf{q}}-2 \Omega \widetilde{\mathbf{G}} \dot{\mathbf{q}}+\left(\widetilde{\mathbf{K}}+\Omega^{2} \widetilde{\mathbf{C}}\right) \mathbf{q}=\mathbf{Q}_{\mathbf{q}}+\Omega^{2} \widetilde{\mathbf{L}},
$$

where $\widetilde{\mathbf{C}}$ is a matrix defined as follows

$$
\widetilde{\mathbf{C}}=\widetilde{\mathbf{K}}+\Omega^{2}\left(\widetilde{\mathbf{J}} \widetilde{\mathbf{J}}^{T}-\widetilde{\mathbf{G}} \widetilde{\mathbf{G}}^{T}-\widetilde{\mathbf{E}}\right) .
$$

The physical meanings of the terms of the equation (26) are:

$$
\begin{array}{ll}
\mathrm{Q}_{\mathrm{q}} & \begin{array}{l}
\text { Generalised external forces } \\
\widetilde{\mathrm{K}}_{\mathrm{q}}
\end{array} \\
\ddot{\mathrm{q}} & \begin{array}{l}
\text { Elastic forces } \\
\text { Acceleration component due to } \\
\text { deformation }
\end{array} \\
-2 \Omega \widetilde{\mathrm{K}} \dot{\mathrm{q}} & \begin{array}{l}
\text { Gyroscopic term } \\
\Omega^{2} \widetilde{\mathrm{C}} \mathrm{q}-\Omega^{2} \widetilde{\mathrm{L}}
\end{array} \\
& \begin{array}{l}
\text { Centripetal acceleration due to } \\
\text { rotation. The former term is } \\
\text { associated with the deformation } \\
\text { of the solid. The latter, with the } \\
\text { undeformed shape }
\end{array}
\end{array}
$$

Since the external forces are always applied in the same spatial points, the term of the generalised force can be reformulated as follows

$$
\begin{aligned}
\mathbf{Q}_{\mathbf{q}}(t)= & \mathbf{B} \int_{\text {Volume }} \boldsymbol{\Phi}\left(\mathbf{u}^{\prime}\right)^{\mathrm{T}} \mathbf{A}^{\mathrm{T}} \mathbf{f}\left(\mathbf{A} \mathbf{u}^{\prime}, t\right) d v= \\
& \int_{\text {Volume }} \boldsymbol{\Phi}(\mathbf{u})^{\mathrm{T}} \mathbf{f}(\mathbf{u}, t) d v= \\
& \int_{\text {Volume }}^{\text {Tolume }}(\mathbf{u})^{\mathrm{T}} \mathbf{f}_{u}(\mathbf{u}) d v \mathbf{f}_{t}(t),
\end{aligned}
$$


and defining $\widetilde{\mathrm{F}}$ as

$$
\widetilde{\mathbf{F}}=\int_{\text {Volume }} \boldsymbol{\Phi}(\mathbf{u})^{\mathrm{T}} \mathbf{f}_{u}(\mathbf{u}) d v,
$$

the generalised forces can be written in this way

$$
\mathbf{Q}_{\mathbf{q}}(t)=\widetilde{\mathbf{F}} \mathbf{f}_{t}(t) .
$$

The main advantage of formulating the equation of motion in Eulerian coordinates lies in the fact that matrices $\widetilde{\mathrm{G}}, \widetilde{\mathbf{K}}, \widetilde{\mathrm{C}}, \widetilde{\mathbf{F}}$ and $\tilde{\mathrm{L}}$ do not depend on time and consequently, they can be computed at the beginning of the simulation.

\subsection{Computational method}

The modal properties of the railway wheelset are obtained through a FE model. Thus the matrix $\boldsymbol{\Phi}_{\mathrm{FE}}$ is calculated for the nodes of the mesh. The modal shape function matrix can be estimated interpolating the nodal values

$$
\boldsymbol{\Phi}(\mathbf{u})=\left(\sum_{e=1}^{N_{E}} \mathbf{N}_{\mathbf{e}}(\mathbf{u})\right) \boldsymbol{\Phi}_{\mathrm{FE}},
$$

being $\mathbf{N}_{e}(\mathbf{u})$ the shape function of the element $e$.

Replacing (31) in equations (5) to (7), (25) and (29), a set of matrix expressions are obtained through the integrals of the shape functions

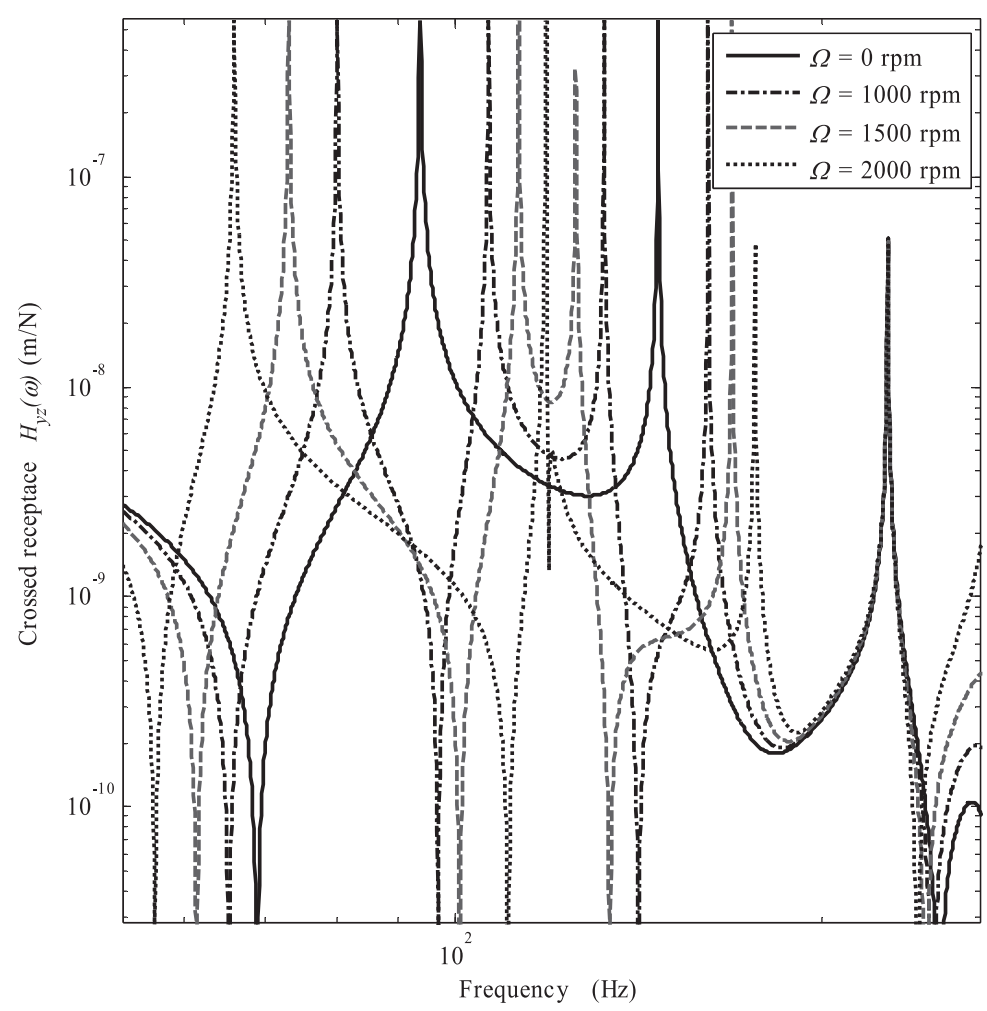

Figure 4. Crossed receptance modulus $H_{y z}(\omega)$ calculated for different angular velocities of the wheelset.

$$
\begin{array}{r}
\widetilde{\mathbf{J}}=\boldsymbol{\Phi}_{\mathrm{FE}}^{\mathrm{T}}\left(\sum_{e=1}^{N_{E}} \int_{V_{e}} \rho \mathbf{N}_{\mathbf{e}}(\mathbf{u})^{\mathrm{T}} \mathbf{J} \mathbf{N}_{\mathbf{e}}(\mathbf{u}) d v\right) \boldsymbol{\Phi}_{\mathrm{FE}}, \quad(32) \\
\widetilde{\mathbf{G}}=\boldsymbol{\Phi}_{\mathrm{FE}}^{\mathrm{T}}\left(\sum_{e=1}^{N_{E}} \int_{V_{e}} \rho\left(\sum_{i=1}^{3} \frac{\partial \mathbf{N}_{e}(\mathbf{u})^{\mathrm{T}}}{\partial v_{i}}(\mathbf{J} \mathbf{u})_{i}\right) \mathbf{N}_{e}(\mathbf{u}) d v\right) \boldsymbol{\Phi}_{\mathrm{FE}},
\end{array}
$$

$$
\begin{aligned}
\widetilde{\mathbf{E}}=\boldsymbol{\Phi}_{\mathrm{FE}}^{\mathrm{T}}\left(\sum_{e=1}^{N_{E}} \int_{V_{e}} \rho \mathbf{N}_{e}(\mathbf{u})^{\mathrm{T}} \mathbf{E} \mathbf{N}_{e}(\mathbf{u}) d v\right) \boldsymbol{\Phi}_{\mathrm{FE}} \\
\widetilde{\mathbf{F}}=\boldsymbol{\Phi}_{\mathrm{FE}}^{\mathrm{T}}\left(\sum_{e=1}^{N_{E}} \int_{V_{e}} \mathbf{N}_{e}(\mathbf{u})^{\mathrm{T}} \mathbf{f}(\mathbf{u}) d v\right) \\
\widetilde{\mathbf{L}}=\boldsymbol{\Phi}_{\mathrm{FE}}^{\mathrm{T}}\left(\sum_{e=1}^{N_{E}} \int_{V_{e}} \rho \mathbf{N}_{e}(\mathbf{u})^{\mathrm{T}} \mathbf{E} \mathbf{u} d v\right)
\end{aligned}
$$

where $N_{E}$ is the number of elements of the mesh and $V_{e}$ is the volume of the $e-$ th element.

\section{Results}

The first result presented in Figure 4 corresponds to the Frequency Response Function (FRF) crossed receptance: the force is applied in the contact point, in the vertical direction ( $z$-axis); the displacement is measured in the transversal direction ( $y$-axis, lateral direction to the track axis). The receptance expression can be obtained from (26) as follows

$\mathbf{H}(\omega)=\boldsymbol{\Phi}^{\mathrm{T}}\left[-\omega^{2} \mathbf{I}-i 2 \omega \Omega \widetilde{\mathbf{G}}+\widetilde{\mathbf{K}}+\Omega^{2} \widetilde{\mathbf{C}}\right]^{-1} \boldsymbol{\Phi}$,

where $i$ is the imaginary unit and $\mathbf{I}$ is the identity matrix. Figure 4 shows in continuous black trace the FRF for the non-rotating wheelset. In the frequency range shown (from 53 to 270 $\mathrm{Hz}$ ), the curve presents three resonances at 93.7 $\mathrm{Hz}$ (1st bending mode), $146.8 \mathrm{~Hz}$ (2nd bending mode) and $226.7 \mathrm{~Hz}$ (umbrella mode, see Figure 2 ). These results can be foreseen through the Classical Vibration Theory.

If the angular velocity of the wheelset is non-zero, the peaks of the FRF associated with modes with multiplicity 2 produce two peaks that can be interpreted as the forward and backward whirl modes [2] in rotating beams. At $\Omega=1000 \mathrm{rpm}$ (200 km/h aprox.) the backward mode frequency is $87 \mathrm{~Hz}$ and the forward one is $107 \mathrm{~Hz}$. It must be highlighted that modes with multiplicity 1 do not split into two peaks.

Taking into account the symmetry of revolution of the solid, the response of the 
contact point of a nonrotating wheelset in the longitudinal direction ( $x$-axis, or track axis direction) will be zero if the excitation is produced in the vertical direction. However, Figure 5 shows non-null crossed receptance $H_{x z}(\omega)$ calculated for the rotating wheelset. The level of the response is similar to that in the transversal direction. It can be observed in Figure 6, where the graphic shows the orbit of the contact point if a harmonic vertical force is applied in the same point. The excitation frequency $(87 \mathrm{~Hz})$ is very close to the backward whirl mode associated with the 1 st bending mode of the solid.

The FRF mobility, that is

$$
\mathbf{Y}(\omega)=i \omega \mathbf{H}(\omega)
$$

provides a clearer relation with the wear mechanism that trigger the corrugation. Figure 7 presents the mobility $Y_{x z}(\omega)$, where the velocity is measured in the longitudinal direction and the harmonic force is applied vertically.

\section{Conclusions}

Most of corrugation cases that take place in the railway tracks are related to an adverse coupling between the wear mechanism of the rails and the dynamics of the unsprung masses of the vehicle and the track. In order to estimate the corrugation pattern, some authors propose to calculate the wear depth from the results of the train-track dynamic simulation (see example in Ref. [17]). However, none of the developed methodologies take account of a realistic wheelset model that considers its flexibility and the inertial effects due to rotation.

The present article develops a wheelset dynamic model that can be implemented in the simulation of the corrugation growth. The model is based on a general procedure for dynamic of rotating solids of revolution presented in [16]. The analysis of the FRF obtained from the model lets us determine the complex coupling in the spatial domain (dynamic coupling between vertical, lateral and longitudinal forces and displacements in the wheel contact point with the rail) and in the frequency domain (coupling of different modes of the non-rotating wheelset); this coupling is due to the rotation of the solid.

The appearance of the orbits in the contact point let us consider that constant longitudinal forces (braking, motor torque or curving) can excite the backward or the forward mode. The results open the possibility of self-excited dynamics that can set off the corrugation process.

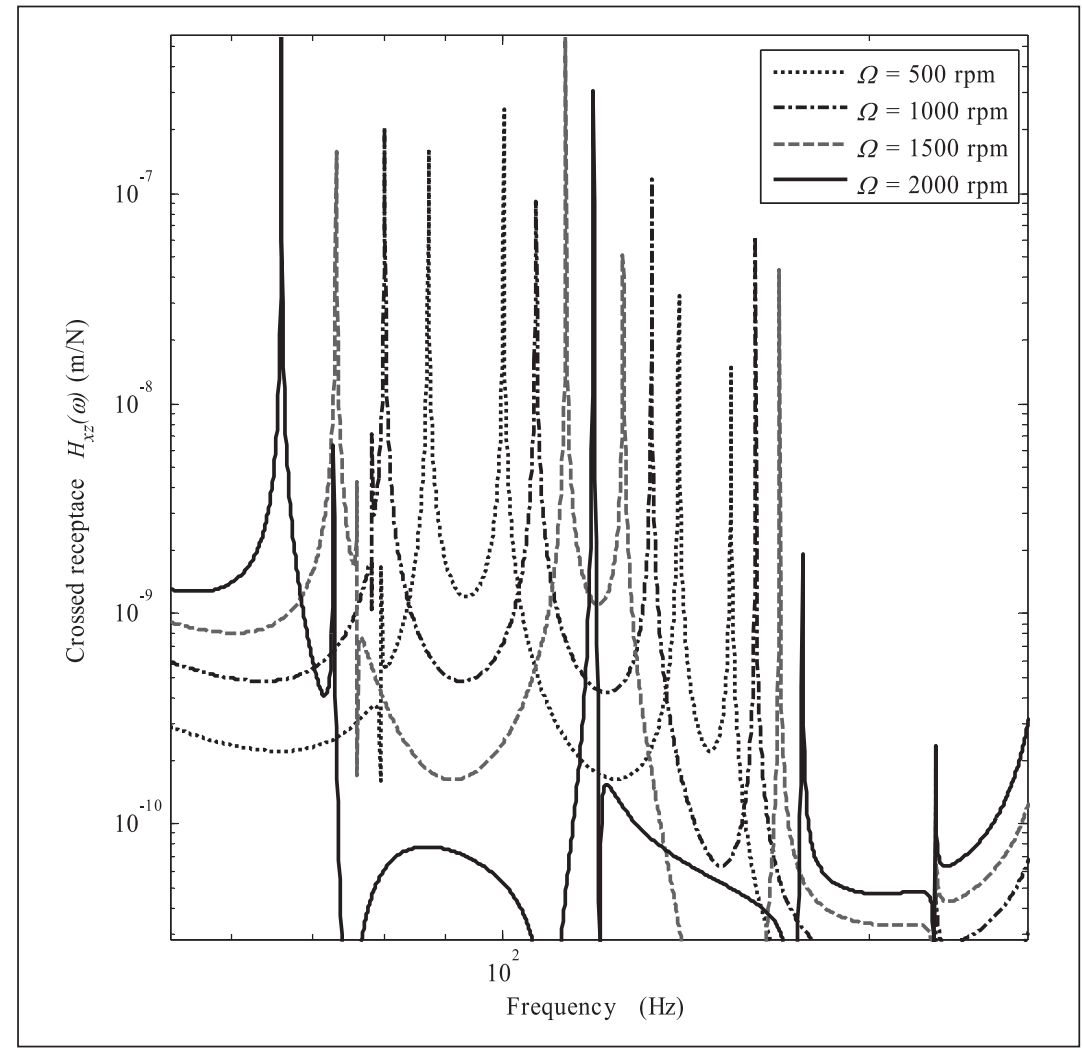

Figure 5. Crossed receptance modulus $H_{y z}(\omega)$ calculated for different angular velocities of the wheelset.

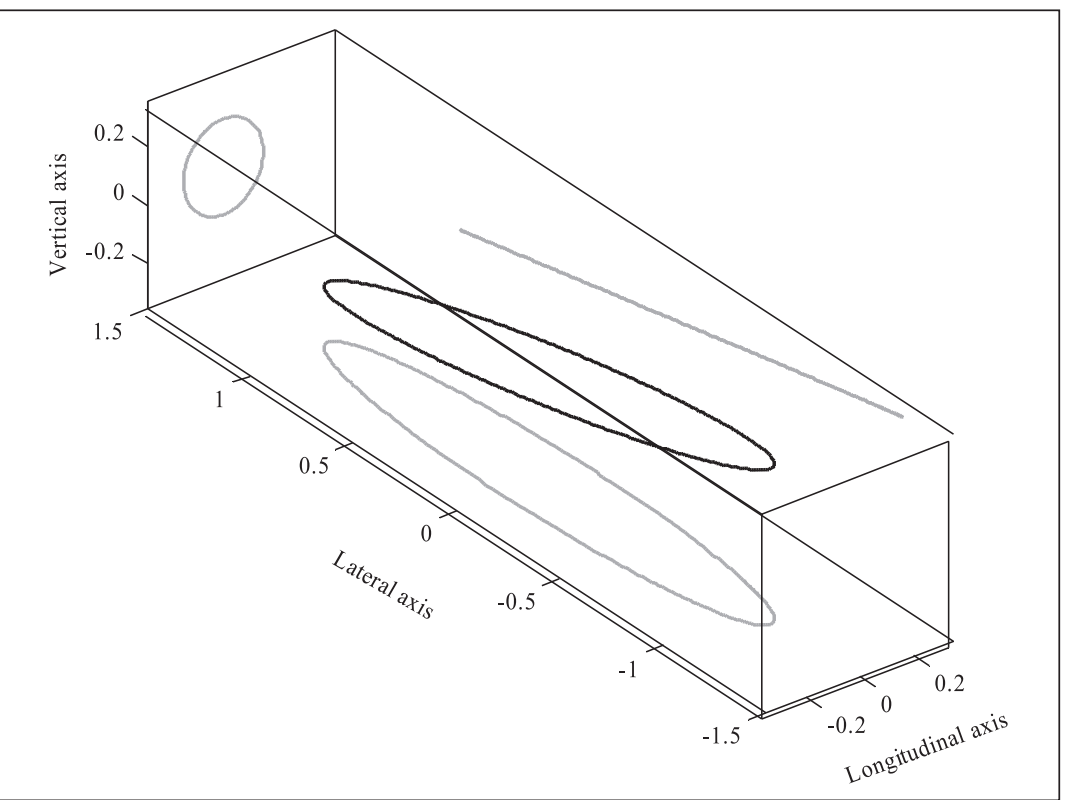

Figure 6. In black trace, orbit of the contact point if a vertical excitation is applied. The angular velocity of the wheelset is $\Omega=500 \mathrm{rpm}(100 \mathrm{~km} / \mathrm{h}$ aprox). The excitation frequency is $87 \mathrm{~Hz}$. In grey trace, the projections on the orthogonal planes of the orbit are drawn.

\section{Acknowledgements}

This work was sponsored by Projects T79/2006 (Ministerio de Fomento - Metro de Madrid- CDM) and TRA2007-67167 (Ministerio de Educación y Ciencia - FEDER). 


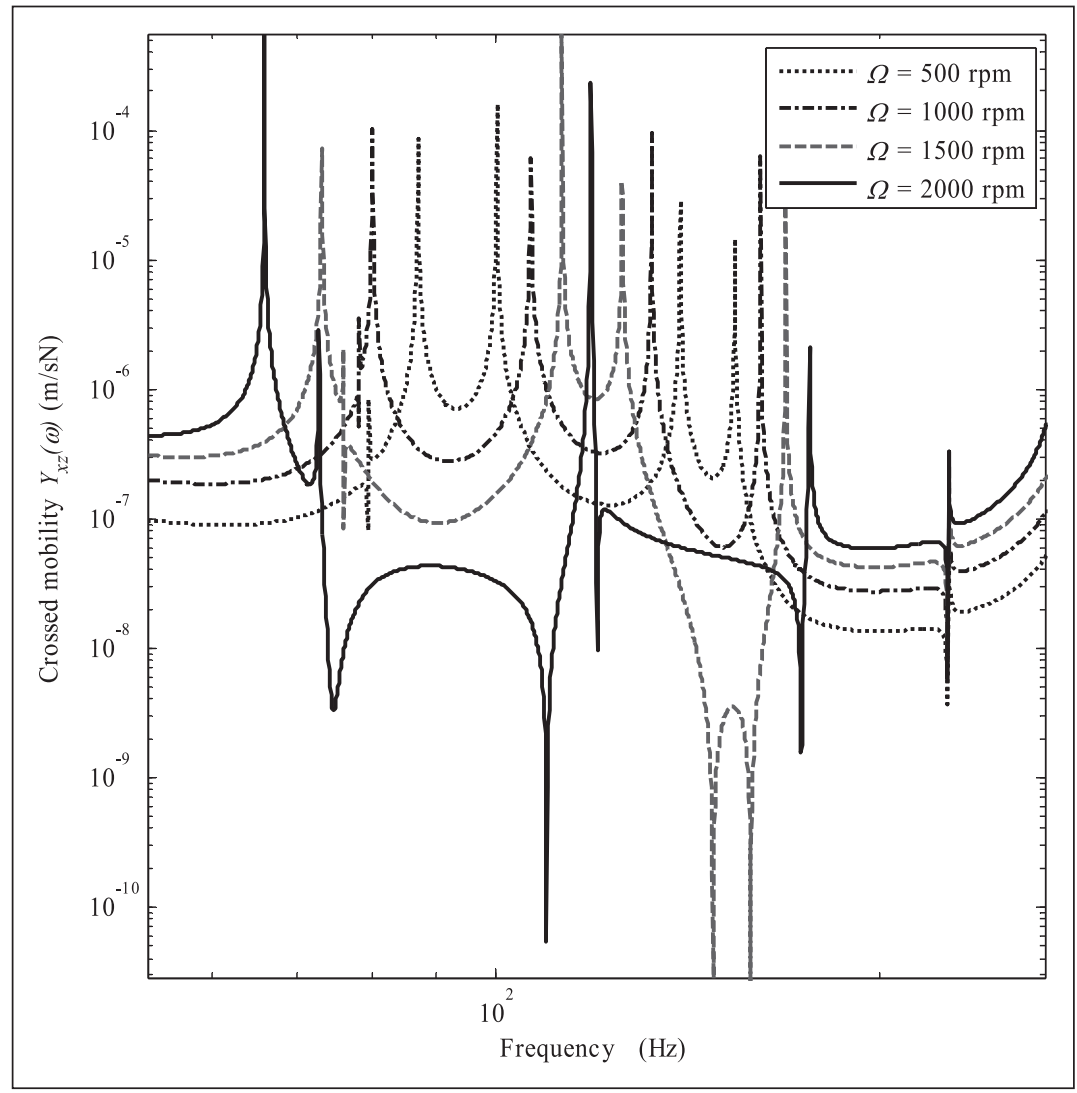

Figure 7. Crossed mobility modulus $Y_{y z}(\omega)$ calculated for different angular velocities of the wheelset.

\section{References}

[1] D. J. Thompson, Wheel-rail noise generation, part V: inclusion of wheel rotation, Journal of Sound and Vibration, Vol. 161, No 3 (1993), pp. 467-482.

[2] J. Fayos, A. Rovira, L. Baeza, J. Carballeira, Rotating shaft analytical response in adimensional form, In Proceedings of the 13th International Congress on Sound and Vibration, Vienna (2006).

[3] T. Szolc, Medium frequency dynamic investigation of the railway wheelsettrack system using a discrete-continuous model, Archive of Applied Mechanics, Vol. 68 (1998), pp. 30-45.

[4] T. Szolc, Simulation of bendingtorsional-lateral vibrations of the railway wheelset-track system in the medium frequency range, Vehicle System Dynamics, Vol. 30, No. 6 (1998), pp. 473-508.

[5] T. Szolc, Simulation of dynamic interaction between the railway bogie and the track in the medium frequency range, Multibody System Dynamics, Vol. 6 (2001), pp. 99-122.

[6] H. Claus, W. Schiehlen, Dynamic stability and random vibrations of rigid and elastic wheelsets, Nonlinear Dynamics, Vol. 36 (2004), pp. 299-311.

[7] A. A. Shabana, Dynamics of multibody systems, Cambridge University Press (1998).

[8] M. A. Brown, A. A. Shabana, Application of multibody methodology to rotating shaft problems, Journal of Sound and Vibration, Vol. 204 (1997), pp. 439-457.

[9] T. Meinders, Modelling of a railway wheelset as a rotating elastic multibody system, Machine Dynamic Problems, Vol. 20 (1998), pp. 209-219.

[10] T. Meinders, P. Meinke, Rotor dynamics and irregular wear of elastic wheelset, In K. Popp and W. Schiehlen, editors, Lecture Notes in Applied Mechanics, pp. 133-152. Springer, Berlin, 2003.

[11] K. Popp, H. Kruse, I. Kaiser, Vehicletrack dynamics in the mid-frequency range, Vehicle System Dynamics, Vol. 31 (1999), pp. 423-464.

[12] K. Popp, I. Kaiser, H. Kruse, System dynamics of railway vehicles and track, Archive of Applied Mechanics, Vol. 72 (2003), pp. 949-961.

[13] J. Arnold, I. Kaiser, G. Schupp, Simulation of a railway vehicle's running behaviour: How elastic wheelsets influence the simulation results, Vebicle System Dynamics, Vol. 41, Suppl. (2004), pp. 242-251.

[14] Kaiser and K. Popp, Interaction of elastic wheelsets and elastic rails: modelling and simulation, In Proceedings of 19th Symposium of the International Association for Vebicle System Dynamics, Milano (2005).

[15] I. Kaiser, A. Heckmann, F. van der Linden, On an ALE-approach for rotating elastic structures, In Proceedings of ECCOMAS Congress, Milano (2007).

[16] J. Fayos, L. Baeza, F. D. Denia, J. E. 
Tarancón, An Eulerian coordinate-based method for analysing the structural vibrations of a solid of revolution rotating about its main axis, Journal of Sound and Vibration, Vol. 306 (2007) pp. 618-635.
[17] L. Baeza, P. Vila, A. Roda, J. Fayos, Prediction of corrugation in rails using a non-stationary wheelrail contact model, Wear (2008).

doi:10.1016/j.wear.2008.01.024, In Press, available online 19 May 2008.

\section{Noise pollution threatens animals}

Noise pollution is becoming a major threat to the welfare of wildlife, according to a scientific review. Sounds produced by vehicles, oil and gas fields and urban sprawl interfere with the way animals communicate, mate and prey on one another. The sounds are becoming so ubiquitous that they may threaten biodiversity, say the review's authors. Even the animals living in protected National Parks in the US are being exposed to chronic levels of noise. Writing in the journal Trends in Ecology and Evolution, three scientists based in Fort Collins, Colorado, US detail the extent to which noise pollution is now harming wild animals. Dr Jesse Barber and Dr Kevin Crooks of Colorado State University and Dr Kurt Fristrup of the US National Park Service reviewed all recent scientific studies examining the issue. They found that man-made noise is already causing a catalogue of problems. "Many animal species evolved hearing sensitive enough to take advantage of the quietest conditions; their hearing is increasingly compromised by noise," said Dr Barber. That intrusion can have a significant impact on the way wild animals communicate. Great tits (Parsus major) sing at higher frequencies in response to urban noise, so they are better able to hear each other. But not all animals are able to adapt in this way. Female grey tree frogs (Hyla chrysoscelis) exposed to the sounds of passing traffic take longer to locate and find calling males, while European tree frogs (Hyla arborea) call less overall. Crucially, both species appear unable to change their calling habitats to overcome the din from the roads, potentially compromising their ability to reproduce. Noise pollution can also effect the ability of many animals such as owls and bats to find and hunt their prey. Laboratory studies have shown that gleaning bats, which locate prey by the sounds they make, avoid hunting in noisy areas. That can place gleaning bats at a higher risk of extinction, as noise pollution increasingly corrupts once habitable areas. For example, one gleaning bat species, the Bechstein's bat (Myotis bechsteinii), is less likely to cross roads than other bat species that forage in open areas, suggesting the noise of the traffic could fragment their hunting grounds. The bat occurs across Europe including in the south of the UK. In the Amazon, terrestrial insectivores, which also hunt using sound, especially avoid areas where roads are being constructed.

\section{Newport News officials revised noise ordinance}

For almost eight months Newport News has been without a noise ordinance. City staff stopped enforcing the previous ordinance after the state Supreme Court ruled Virginia Beach's ordinance, which was similar to the one used in Newport News, unconstitutional because it was too vague. Now, interim City Manager Neil Morgan has presented a revised ordinance the council might vote on soon. "We want to get something in place that deals with most types of nuisances, and then tweak it as we go along," Morgan said. The largest number of noise complaints are about loud music - either from late-night parties or cars driving by with blaring stereo systems and barking dogs. The new ordinance addresses both. But the old one dealt with those problems, too. The difference, said City Attorney Stuart Katz, is in defining the nuisance. The old ordinance deemed something a nuisance if it bothered a reasonable person. The new ordinance defines nuisance noise by how far and where it travels. Noise might be a nuisance if it can be heard across property boundaries or in the residence of someone else between the hours of 10 p.m. and 8 a.m, or if it can be heard at a distance of 50 feet or more. "The court found fault with the reasonable-person standard." Katz said. Mayor Joe S. Frank was concerned about whether the ordinance addressed moving vehicles with thumping stereos. "Autos are the biggest issue," he said. "I don't see this as enforcement." Morgan said the ordinance can be adjusted, and both he and Katz said moving vehicles are addressed by the definitions of "nuisance." The difficulty has always been catching the vehicle as it is travelling away from the person troubled by the noise, but Katz said if the vehicle is reported and can be stopped, the driver can be cited under the new ordinance. "For 90 percent of the circumstances where there is a legitimate complaint, this gives you a tool," Morgan said. 